\title{
(S,s)-adjustment Strategies and Hedging under Markovian Dynamics
}

\author{
Elettra Agliardi ${ }^{\mathrm{a}, \mathrm{b}}$ and Rainer Andergassen ${ }^{\mathrm{a}, \mathrm{b}}$ \\ ${ }^{a}$ Department of Economics, University of Bologna, Piazza Scaravilli, 2, 40126 Bologna (BO), Italy. \\ E-mail: elettra.agliardi@unibo.it \\ ${ }^{b}$ RCEA, Via Patara, 3, 47900 Rimini (RN), Italy. \\ E-mail: rainer.andergassen@unibo.it
}

We study the destabilizing effect of hedging strategies under Markovian dynamics with transaction costs. Once transaction costs are taken into account, continuous portfolio rehedging is no longer an optimal strategy. Using a non-optimizing (local in time) strategy for portfolio rebalancing, explicit dynamics for the price of the underlying asset are derived, focusing in particular on excess volatility and feedback effects of these portfolio insurance strategies. Moreover, it is shown how these latter depend on the heterogeneity of the insured payoffs. Finally, conditions are derived under which it may be still reasonable, from a practical viewpoint, to implement Black-Scholes strategies. The Geneva Risk and Insurance Review (2011) 36, 112-131. doi:10.1057/grir.2010.7; published online 17 August 2010

Keywords: (S,s)-adjustment strategies; dynamic hedging; volatility; Black-Scholes model; transaction costs

\section{Introduction}

Standard option pricing literature relies on the hypothesis that the dynamics of the underlying asset are independent of the hedging strategy. Dynamic delta hedging strategies and portfolio insurance require to sell the underlying asset if its price decreases, while they require to buy if its price increases. The hypothesis of independence between hedging and price dynamics corresponds to the assumption that the market for the underlying asset is perfectly liquid.

Positive feedback effects from dynamic delta hedging strategies have been studied recently assuming that the market for the underlying asset is only finitely liquid, that is, relaxing one of the major assumptions of the BlackScholes model that the market is perfectly elastic ${ }^{1}$ (see, for example, Gennotte

\footnotetext{
${ }^{1}$ Brennan and Schwartz (1989), on the contrary, study the effects of portfolio insurance on financial markets abstracting from possible liquidity problems. They assume that agents are only concerned with long-term prospects of the assets and do not change their expectations in reaction to changes in current prices. As a result, markets are very liquid and the feedback effects of hedging on volatility become very small.
} 
and Leland, 1990; Donaldson and Uhlig, 1993; Frey and Stremme, 1997; Sircar and Papanicolaou, 1998; Schönbucher and Wilmott, 2000). It has been shown that in this case portfolio insurance activity has a destabilizing effect on the dynamics of the price of the underlying asset. In particular, it increases price volatility. Frey and Stremme (1997) study the feedback effects of dynamic hedging strategies on the volatility of the market equilibrium price. They derive the tracking error, show that an overinvestment is required and derive the best volatility used by programme traders in calculating their trading strategy. Sircar and Papanicolaou (1998) study the interaction between reference traders and programme traders and the feedback effects of programme traders on the underlying asset, deriving a feedback-adjusted option price and the optimal hedging strategy. Frey (1998), in a continuous time version of Jarrow (1992, 1994), study the replication of derivative securities from the viewpoint of large traders whose trades have a non-negligible effect on the asset price. In a similar vein, Schönbucher and Wilmott (2000) discuss the pricing, hedging and replication of options if a larger trader, for whom the market is illiquid, interacts with small traders. Among the contributions related to the role of portfolio insurance strategies some relevant papers have demonstrated the possibility of multiple equilibrium prices in illiquid markets. Gennotte and Leland (1990) show that information differences among market participants can cause markets to be relatively illiquid and discontinuities (or crashes) can occur even with relatively little hedging. Like Gennotte and Leland (1990), Donaldson and Uhlig (1993) show that the existence of atomistic portfolio insurers increase the variance of possible equilibrium prices and can lead to situations with many potential equilibrium prices for a single set of fundamentals, unless large portfolio insurers act in a centralized way.

All the above-mentioned papers assume that programme traders can buy and sell assets without incurring transaction costs. But, as a matter of fact, transaction costs are non-negligible in asset markets. Our paper, which is most closely aligned with Frey and Stremme (1997), extends the literature on market equilibrium models with feedback effects caused by dynamic hedging to the case of transaction costs.

If we introduce transaction costs, then it is no longer optimal to adjust the portfolio continuously. There are two main approaches in the literature taking the effects of transaction costs into account: the first considers discrete portfolio adjustments, where the time step of portfolio rebalancing is exogenously given, while the second considers traders as continuously monitoring the price of the underlying asset, although adjusting their portfolio only if the gain from adjustment is larger than the cost of adjustment. This latter approach can be subdivided into two further approaches: the first is called local in time (Leland, 1985; Hoggard et al., 1994), whereas the second is called global in time (Davis et al., 1993; Whalley and Wilmott, 1997; 
Constantinides and Zariphopoulou, 1999, 2001). The former is a nonoptimizing approach, where rehedging is based on minimizing the variance of the hedged portfolio, while the latter is an optimizing one and is based on utility maximization and stochastic optimal control. The option value is obtained by a comparison of the maximum utilities of trading with and without the obligation of fulfilling the option contract at expiry (for a review see, for example, Wilmott, 2000). In this framework, Davis et al. (1993) consider European option pricing with proportional transaction costs charged on sales and purchases of stock. Whalley and Wilmott (1997) provide an asymptotic analysis of Davis et al. (1993) in the limit of small transaction costs. Constantinides and Zariphopoulou $(1999,2001)$ derive in closed form bounds to the reservation price of a call option for a large class of utility functions.

All these papers are concerned with the pricing of derivatives in the presence of transaction costs, but do not deal with the hedging effects in a market model.

Our paper considers a "finitely liquid" market model and, following a local in time approach, assumes that hedging takes place at flexible stochastic trading periods, instead of fixed-interval times, and that transaction costs are fixed, instead of proportional. ${ }^{2}$

Once transaction costs are taken into account, an appropriate hedging strategy has to be found since it is no longer optimal to re-hedge immediately the portfolio as the asset price changes. In his seminal paper Leland (1985) first introduced proportional transaction costs and developed a pricing model with a modified option replicating strategy depending on the level of transaction costs and the revision interval. Such strategy replicates the option inclusive of transaction costs, with an error that is uncorrelated with the market and is claimed to approach zero as the revision period becomes shorter. Inclusive of transaction costs, the bid-ask spread of the underlying asset becomes larger and the accentuation of up and down movements of the asset price is modelled as if the volatility of the actual asset price is higher. Kabanov and Safarian (1997) calculate the limiting error in Leland's hedging strategy for the approximate pricing of the European call. They partially correct a result in Leland (1985), showing that such limiting error equals zero only when the level of transaction costs decreases to zero as the revision interval tends to zero. Bensaid et al. (1992) deal with a discrete time model with proportional transaction costs and derive optimal portfolios sequentially, finding

\footnotetext{
${ }^{2}$ The model could be extended trivially in order to consider also proportional transaction costs. For a discussion on transaction costs and their empirical estimation see, for example, Hasbrouck (2009).
} 
dominating strategies of the $(\mathrm{S}, \mathrm{s})$-type and a range within which the derivative price should lie, defining its bid-ask spread.

Most above-mentioned papers with transaction costs assume a given fixedrevision interval. In this paper instead we consider a stochastic revision interval. We introduce adjustment hazard functions for each programme trader in a way which is new in this literature. Following Whalley and Wilmott (1993) and Henrotte (1993), we define a confidence level for the deviation of the risky asset position from the perfect hedge such that for each agent inaction is optimal as long as the hedging imbalance level is below a tolerance level $H_{0}$, while the position should be rebalanced once the hedging imbalance level is above $H_{0}$. The value $H_{0}$ gives a measure of the maximum expected risk in the portfolio.

We assume that $H_{0}$ is partly deterministic and partly stochastic: the former captures the influence of transaction costs, such as trading fees, whereas the latter captures stochastic contingencies. Given these assumptions, an adjustment hazard function (that is, the probability that the trader rebalances his position conditional on his imbalance level) for each programme trader can be defined. Following Caballero and Engel (1993, 1999), we study the aggregate dynamics of the adjustment hazard rates. For a given asset price level we calculate the average aggregate demand of the programme traders using the distribution of imbalance levels. Then, the resulting price dynamics of the risky asset are derived and the average size of the adjustment is characterized.

Our main results are in keeping with the literature on increased market volatility from hedging strategies and on the fact that the insurance activity of programme traders together with a finite market liquidity may lead to market destabilization and eventually to a market collapse (see, for example, Gennotte and Leland, 1990). ${ }^{3}$ Our results are consistent with an overestimation of implied volatility when applying the Black-Scholes formulae (see, for example, Rubinstein, 1985; Dumas et al., 1998; Platen and Schweizer, 1998; Cetin et al., 2006) and offer additional insight into the excess volatility puzzle. ${ }^{4}$ As discussed in 'Positive feedback effects from hedging' section, in our model excess volatility is driven by transaction costs, liquidity issues and the aggregate

\footnotetext{
${ }^{3}$ The portfolio insurance trading strategies are often implemented on a large scale and yet the liquidity of their market is sometimes very limited. As in the case of the October 1987 stock market crash, some empirical studies and even official reports (i.e., the Brady Commission Report) suggest that portfolio insurance trading and replicating strategies contribute to aggravate the effects of the crash.

${ }^{4}$ The excess volatility puzzle and some explanations of its possible sources have been discussed in the literature starting from Shiller (1981) and LeRoy and Porter (1981). Most papers focus on heterogeneity of information and beliefs and liquidity issues (see, for example, Le Roy (1989) and Shiller (2003) for reviews).
} 
characteristics of programme traders. In particular, we specify in which way markets are finitely liquid when transaction costs are introduced and the role of transaction costs in determining the size and the frequency of adjustment. Our paper provides results that are consistent with the approach of Cetin et al. (see, for example, Cetin et al., 2004; Cetin et al., 2006; Cetin et al., 2007), although they do not deal with models of feedback effects. Instead of concentrating on the feedback effects that govern the dependence of the equilibrium stock price on the portfolio actions of programme traders, as we do in our paper, they have a reduced form illiquidity model, introducing a supply curve which excludes strategic trading and where liquidity costs are increasing in the position to liquidate. One major result of Cetin et al. is that the continuous trading strategies of arbitrarily small quantities incur no liquidity costs, while trading and also optimal hedging in discrete time produce a non-zero liquidity premium for options, which is also confirmed in their empirical work (see Cetin et al., 2006).

Finally, our paper has some relevance in an insurance context too. Put replication trading is often used in insurance contracts. Many insurance contracts that are used today are derived from replicating strategies for derivative securities. The destabilizing effects may be especially strong in markets with low liquidity. This is often the case of insurance markets and financial markets dealing with products with an insurance content (for example, see Gibson et al., 2008). A model like ours provides a further contribution explaining the destabilizing effects that may emerge in markets with low liquidity.

The paper is organized as follows. In 'The basic model' section, the adjustment hazard function is formally introduced and the model is presented. 'Positive feedback effects from hedging' section contains the main results. Finally, 'Conclusion' section concludes.

\section{The basic model}

Suppose that there are two types of traders operating in a market, where there is a risky asset and a riskless one (a pure discount bond): programme traders and reference traders. Programme traders use a dynamic hedging strategy, whereas reference traders are small price takers, which include market makers and market timers, providing liquidity for market transactions. In what follows, we do not model the reference traders' investment problem explicitly, but rather their aggregate behaviour. We assume that there is a continuum of reference traders, such that the effects of transaction costs on the aggregate demand function of the reference traders are negligible. The reference traders are unaware of the programme traders' presence and trading strategy (so that 
we can avoid strategic trading). Furthermore, we suppose that reference traders have perfect information about the fundamentals of the risky asset. The aggregate demand function of the reference traders is denoted by $D\left(t, F_{t}, S_{t}\right)$, where $S$ is the price of the risky asset, $t$ is time and $F$ can be interpreted in different ways. For example, Frey and Stremme (1997) and Sircar and Papanicolaou (1998) assume $F$ to be the aggregate income of the reference traders, whereas Platen and Schweizer (1994) assume $F$ to be an unspecified liquidity demand, and others assume $F$ to be the fundamental value of the firm. We follow this latter approach, and make the following assumptions about the aggregate demand of the reference traders:

\section{Assumption 1}

(a) $D \in C^{2}:[0, \infty) \times \mathbb{R}_{+}^{2} \rightarrow \mathbb{R}$;

(b) there exists a sufficiently large $d>0$ such that $\partial D / \partial S \leqslant-d$;

(c) $\partial D / \partial F>0$;

(d) for any $t, F, \lim _{S \rightarrow+\infty} D(t, F, S)=0, \lim _{S \rightarrow 0^{+}} D(t, F, S)=+\infty$

Assumption 1(b) indicates that as the asset price increases, demand decreases, so that, everything else being equal, the reference traders would like to hold more assets if the price is low and fewer if it is high. Economically, ruling out the derivative to be zero means that demand does react to price changes; otherwise it would not be possible to find an equilibrium by adjusting the price and the market would be illiquid. Moreover, it is required that demand sufficiently reacts to price changes, as will become clear in 'Positive feedback effects from hedging' section (see Proposition 2). Assumption 1(c) means that as the fundamental value of the asset increases, demand increases as well. ${ }^{5}$

Let us normalize the total supply of the risky asset to one. Thus, in the absence of programme traders, equilibrium is guaranteed by the following market clearing condition

$$
D\left(t, F_{t}, S_{t}^{*}\right)=1 .
$$

For every pair $\left(t, F_{t}\right)$ Eq. (1) has exactly one solution in $S_{t}$ denoted by $\varphi\left(t, F_{t}\right)$. Existence is guaranteed by Assumptions 1(a) and 1(d); uniqueness follows from strict monotonicity of $D$ with respect to $S$, which is guaranteed by Assumption 1(b). Assumption 1(b) together with the implicit function theorem implies that $\varphi$ is smooth. Thus, the equilibrium asset price, that is $S_{t}^{*}=\varphi\left(t, F_{t}\right)$, is a function of its fundamental value. We call $S_{t}^{*}$ the normal price.

\footnotetext{
${ }^{5}$ Assumption 1 could be relaxed, following a compactness argument, where the above properties are satisfied by a suitable sequence of $D^{n}(),. n=1,2 \ldots$ converging uniformly to $D$ (see also Frey and Stremme, 1997).
} 
We assume that the dynamics of the fundamental value of the risky asset follow a diffusion process of the form

$$
d F_{t}=\mu_{F}\left(F_{t}, t\right) d t+\sigma_{F}\left(F_{t}, t\right) d W_{t},
$$

where $W_{t}$ denotes a standard Wiener process. The assumption of Markovian dynamics for $F$ is quite standard in this literature (see, for example, Frey and Stremme, 1997). Given the market clearing condition (1) and the stochastic process for the fundamental value (2) we can determine the dynamics of the asset price $S_{t}^{*}$. We follow Platen and Schweizer (1998) in deriving the dynamics of $S_{t}^{*}$. Applying Ito's Lemma to the equilibrium asset price $S_{t}^{*}=\varphi\left(t, F_{t}\right)$ we have that

$$
d S_{t}^{*}=\varphi_{t} d t+\varphi_{F} d F_{t}+\frac{1}{2} \varphi_{F F}\left(d F_{t}\right)^{2}
$$

For each value of $f$ and $t, s=\varphi(t, f)$, and $D(t, f, \varphi(t, f))=1$ hold. Consequently, using the implicit function theorem we ${ }_{2}$ obtain $\varphi_{t}=-D_{t} / D_{s}$, $\varphi_{F}=-D_{F} / D_{S}$ and $\varphi_{F F}=-\frac{1}{D_{S}}\left[D_{F F}-2 D_{F S} \frac{D_{F}}{D_{S}}+D_{S S}\left(\frac{D_{F}}{D_{S}}\right)^{2}\right]$. Substituting these results into (3), we obtain, after using (2) and rearranging terms,

$$
d S_{t}^{*}=\mu_{S}\left(S_{t}^{*}, t\right) d t+\sigma_{S}\left(S_{t}^{*}, t\right) d W_{t},
$$

where

$$
\begin{gathered}
\sigma_{S}\left(S_{t}^{*}, t\right)=-\sigma_{F}\left(F_{t}, t\right) \frac{D_{F}}{D_{S}} \\
\mu_{S}\left(S_{t}^{*}, t\right)=-\frac{1}{D_{S}}\left[D_{t}+D_{F} \mu_{F}\left(F_{t}, t\right)+\frac{1}{2} D_{S S}\left(\sigma_{F}\left(F_{t}, t\right) \frac{D_{F}}{D_{S}}\right)^{2}\right. \\
\left.+\frac{1}{2} D_{F F}\left(\sigma_{F}\left(F_{t}, t\right)\right)^{2}-D_{S F}\left(\sigma_{F}\left(F_{t}, t\right)\right)^{2} \frac{D_{F}}{D_{S}}\right]
\end{gathered}
$$

In the next section, we are going to study the programme traders' aggregate demand. Then, this aggregate demand is added to the reference traders' demand in (1) and the implications for the dynamics of the risky asset price are studied.

\section{Aggregate demand of programme traders}

Let us first specify the hedging strategy of a single programme trader whose portfolio consists of a European option with strike price $K$ and time to maturity $\tau$ and a position on $G$ underlying assets. Later on, we aggregate the 
hedging strategies among different strike prices and times to maturity. ${ }^{6} \mathrm{We}$ distinguish between two types of volatilities: the input volatility $\sigma$ and the equilibrium market volatility $\sigma_{S}($.). The former is used by programme traders to compute hedging strategies, whereas the latter emerges as an equilibrium (market-clearing) outcome. In this section, we describe the programme traders' aggregate demand and therefore input volatility $\sigma$ is used, whereas in 'Positive feedback effects from hedging' section a consistency condition is derived where the input volatility $\sigma$ coincides with the endogenously found equilibrium market volatility $\sigma_{S}($.$) . Given the input volatility \sigma$, we denote by $V(S, \sigma, \tau, K)$ the option value, where $S$ is the current underlying asset price, whose returns follow a geometric Brownian motion with drift $\mu$. Let $\eta=\eta(S, \sigma, \tau, K)=\Delta(S, \sigma, \tau, K)-G$, with $\Delta(S, \sigma, \tau, K)=\partial V(S, \sigma, \tau, K) / \partial S$, where $\eta$ is called the hedging imbalance. ${ }^{7}$

In case of no transaction costs, continuous adjustment of the portfolio is optimal. We consider here transaction costs in the form of fixed costs of adjustment, where continuous adjustment of the portfolio is no longer optimal. A portfolio adjustment is assumed to occur only if the hedging imbalance $\eta$ exceeds a pre-determined tolerance level, in which case a perfect hedge is established. This adjustment strategy is called in the literature an $(\mathrm{S}, \mathrm{s})$-strategy, ${ }^{8}$ where $\eta$, once hitting a pre-defined threshold, jumps to 0 . In the present context, we consider a stochastic threshold $H_{0}(\xi, c)$, which is the tolerance level for $|\eta|$. In $H_{0}(\xi, c), c \geqslant 0$ is the deterministic component, which captures the influence of the size of the transaction costs on the confidence level, while $\xi$ is the stochastic component which captures the influence of stochastic contingencies on the confidence level (these may also include the stochastic component of transaction costs; see, for example, Leland, 1985). Thus, at time $t$, given the realization of $\xi_{t}$, the trader rebalances his position if his imbalance level $\left|\eta_{t}\right|$ is larger than the threshold $H_{0}\left(\xi_{t}, c\right)$.

Since the tolerance level is stochastic, the adjustment depends on the realizations of these contingencies and therefore the hedging strategy can be described in terms of probabilities. More formally, given $\eta_{t}$ and assuming that the probability distribution of $\xi_{t}$ is i.i.d. for each programme trader and

${ }^{6}$ Observe that a single programme trader may possess a portfolio consisting of a mixture of European options with different strike prices and times to maturity and this is taken into account in the aggregation procedure.

${ }^{7}$ Note that we characterize the programme traders' demand function in terms of deviations from the delta-hedging strategy, which is independent of the drift $\mu$. Therefore, the programme traders' demand is unaffected by $\mu$.

${ }^{8}$ For a discussion of the basic problem see Dixit (1993). 
120

independent of time (see Caballero and Engel, 1999), we can write the probability of adjusting the portfolio at time $t$ :

$$
h\left(\eta_{t}, c\right)=\operatorname{Pr}\left[H_{0}\left(\xi_{t}, c\right) \leqslant\left|\eta_{t}\right|\right]
$$

$h($.$) is the adjustment hazard function and depends on the hedging imbalance$ level $\eta$ and on the level of the deterministic component $c$. We make the following assumptions about $h(\eta, c)$ :

\section{Assumption 2}

(a) $h(\cdot)$ is a smooth function;

(b) $h_{\eta}$ and $h_{\eta \eta}$ are bounded and $h_{\eta}(\eta, c) \geqslant 0$;

(c) $h_{c}(\eta, c)<0$;

(d) $\lim _{c \rightarrow 0} h(\eta, c)=1, \lim _{c \rightarrow 0} h_{\eta}(\eta, c)=0$ and $\lim _{c \rightarrow \infty} h(\eta, c)=0$.

Assumption 2(b) gives technical conditions that will be used in 'Positive feedback effects from hedging' section and states that the probability of adjustment does not decrease as the hedging imbalance level increases; Assumption 2(c) states that the probability of adjustment increases as the size of the transaction costs decreases. Assumption 2(d) implies that, if the size of the transaction costs is vanishing small, then we have continuous adjustment or dynamic delta hedging, whereas, if the transaction costs are infinitely large, then the probability of portfolio adjustment becomes vanishing small.

The hedging imbalance level $\eta$ has a common element for each programme trader, which is the price of the underlying asset $S$, while there are also idiosyncratic components such as the strike price $K$ and the time to maturity $\tau$. Thus, as the price of the underlying asset changes, the hedging imbalance level for each programme trader changes as well, while the way it changes depends on the distribution of strike prices and times to maturity.

To obtain the programme traders' aggregate demand we need to aggregate the hedging imbalance levels across traders using a cross-sectional distribution. At time $t$, given the asset price $S_{t}$ and assuming that all traders use the same input volatility, ${ }^{9}$ traders' imbalances differ in $K$ and $\tau$. We are going to assume a continuous and random influx and outflux of programme traders from the asset market, so that the average composition of payoffs to be replicated remains almost constant over time. Thus, we have that the average demand of

\footnotetext{
${ }^{9}$ This assumption is justified since later on we calculate the input volatility in a consistent way.
} 
programme traders at time $t$ is given by

$$
\Psi(S, \sigma, c)=\int_{\Re_{+}^{2}} \eta(S, \sigma, \tau, K) h(\eta(S, \sigma, \tau, K), c) v(d K \otimes d \tau),
$$

where $v$ has a smooth density function with respect to a Lebesgue-measure and represents the distribution of strike prices $K$ and times to maturity $\tau$ in the portfolio. Thus, expression (6) represents the demand for the underlying asset of the programme traders at time $t$. We are interested in showing how this demand changes as the price of the underlying asset changes.

Consider a change in the price of the risky asset of size $d S$. Using expression (6) we have:

$$
\begin{aligned}
\Psi(S+d S, \sigma, c)= & \int_{\Re_{+}^{2}}[\Delta(S+d S, \sigma, \tau, K)-\Delta(S, \sigma, \tau, K)+\eta(S, \sigma, \tau, K)] \\
& \times h(\Delta(S+d S, \sigma, \tau, K)-\Delta(S, \sigma, \tau, K)+\eta(S, \sigma, \tau, K), c) \\
& \times v(d K \otimes d \tau) .
\end{aligned}
$$

Taking Taylor expansion of $\Delta(S+d S)$ and $h(\Delta(S+d S)-\Delta(S)+\eta(S), c)$ around $S$ and $\eta$ respectively, we have

and

$$
\Delta(S+d S)-\Delta(S)=\Delta_{S} d S+\frac{1}{2} \Delta_{S S}(d S)^{2},
$$

$$
\begin{aligned}
h(\Delta(S+d S)-\Delta(S)+\eta, c)= & h(\eta, c)+h_{\eta}(\eta, c)\left(\Delta_{S} d S+\frac{1}{2} \Delta_{S S}(d S)^{2}\right) \\
& +\frac{1}{2} h_{\eta \eta}(\eta, c)\left(\Delta_{S} d S+\frac{1}{2} \Delta_{S S}(d S)^{2}\right)^{2}
\end{aligned}
$$

Now we can calculate $d \Psi(S, \sigma, c)=\Psi(S+d S, \sigma, c)-\Psi(S, \sigma, c)$ as follows:

$$
\begin{aligned}
& d \Psi(S, \sigma, c)=\int_{\Re_{+}^{2}}\left\{[ \Delta _ { S } d S + \frac { 1 } { 2 } \Delta _ { S S } ( d S ) ^ { 2 } ] \left[h(\eta, c)+h_{\eta}(\eta, c)\right.\right. \\
& \left.\quad \times\left(\Delta_{S} d S+\frac{1}{2} \Delta_{S S}(d S)^{2}\right)+\frac{1}{2} h_{\eta \eta}(\eta, c)\left(\Delta_{S} d S+\frac{1}{2} \Delta_{S S}(d S)^{2}\right)^{2}\right] \\
& \left.\quad+\eta h_{\eta}(\eta, c)\left(\Delta_{S} d S+\frac{1}{2} \Delta_{S S}(d S)^{2}\right)+\frac{1}{2} \eta h_{\eta \eta}(\eta, c)\left(\Delta_{S} d S+\frac{1}{2} \Delta_{S S}(d S)^{2}\right)^{2}\right\} \\
& \quad \times v(d K \otimes d \tau) .
\end{aligned}
$$


Since $(d S)^{\theta}=0$ for $\theta>2$ we obtain, after rearranging terms

$$
\begin{aligned}
d \Psi(S, \sigma, c)=d S & \int_{\Re_{+}^{2}}\left[h(\eta, c)+\eta h_{\eta}(\eta, c)\right] \Delta_{S} v(d K \otimes d \tau) \\
& +\frac{1}{2}(d S)^{2} \int_{\Re_{+}^{2}}\left\{\left[h(\eta, c)+\eta h_{\eta}(\eta, c)\right] \Delta_{S S}\right. \\
& \left.+\left[2 h_{\eta}(\eta, c)+\eta h_{\eta \eta}(\eta, c)\right]\left(\Delta_{S}\right)^{2}\right\} v(d K \otimes d \tau) .
\end{aligned}
$$

Let us introduce the following technical assumption:

Assumption 3 There exist a function $v_{1}$ such that $v(d K \otimes d \tau)=v_{1}(K, \tau) d K d \tau$ where $v_{1}$ has a smooth density function with respect to a Lebesgue measure; $v_{1}$ has a compact support in $R_{+} \times[0, \infty)$.

Assumption 3 implies that the distribution of strike prices/times to maturity and, consequently, the distribution of hedging imbalance levels are relatively heterogeneous. It will become clear that under Assumption 3, the feedback effects on market volatility remain finite (see Proposition 2). Using Assumption 3 we can write

$$
d \Psi(S, \sigma, c)=\Psi_{S}(S, \sigma, c) d S+\frac{1}{2} \Psi_{S S}(S, \sigma)(d S)^{2},
$$

where

$$
\begin{gathered}
\Psi_{S}(S, \sigma, c)=\int_{\Re_{+}^{2}} \tilde{h}(\eta(S, \sigma, K, \tau), c) \Delta_{S}(S, \sigma, K, \tau) v_{1}(K, \tau) d K d \tau \\
\Psi_{S S}(S, \sigma, c)=\int_{\Re_{\Re_{+}^{2}}}\left\{\tilde{h}(\eta(S, \sigma, K, \tau), c) \Delta_{S S}+\tilde{h}_{\eta}(\eta(S, \sigma, K, \tau), c)\left(\Delta_{S}\right)^{2}\right\} \\
\times v_{1}(K, \tau) d K d \tau,
\end{gathered}
$$

and where $\tilde{h}(\eta, c)=\partial \eta h(\eta, c) / \partial \eta=h(\eta, c)+\eta h_{\eta}(\eta, c)$ and $\tilde{h}=\partial \tilde{h}(\eta, c) / \partial \eta=$ $2 h_{\eta}(\eta, c)+\eta h_{\eta \eta}(\eta, c) \cdot \tilde{h}(\eta(S, \sigma, K, \tau), c)$ indicates the change in the expected size of adjustment induced by a change in the asset price $S . \Delta_{S}(S, \sigma, K, \tau)$ is known in the option pricing literature as the parameter gamma, and it indicates, in the absence of transaction costs, how often a position must be rehedged in order to maintain a delta-neutral position. Thus, $\Psi_{S}(S, \sigma, c)$ indicates the average 
adjustment, given a change in the price of the risky asset. $\Psi_{S}(S, \sigma, c)$ depends: (a) on the average size of adjustment, and thus on the properties of the adjustment hazard function and the distribution of imbalance levels (i.e. $v_{1}(K, \tau)$ ); and (b) on the frequency of adjustment, which depends on the average sensitivity of delta with respect to the price. Notice that Assumption 2 implies that $\lim _{c \rightarrow 0} \Psi_{S}(S, \sigma, c)=\int_{\Re^{2}} \Delta_{S}(S, \sigma, K, \tau) v_{1}(K, \tau) d K d \tau=\tilde{\Gamma}(S, \sigma)$, which is the average value of the gamma, while $\lim _{c \rightarrow \infty} \Psi_{S}(S, \sigma, c)=0$. Therefore, if the size of the transaction costs is vanishing small, then $\Psi_{S}(S, \sigma, c)$ converges towards the average value of the gamma and so we are back to the case of dynamic hedging strategies, while if transaction costs are very large, then no portfolio adjustment occurs at all.

\section{Positive feedback effects from hedging}

As we pointed out before, reference traders have perfect information about the fundamental value of the risky asset. Thus, a reduction in the fundamental value leads to a decrease in the price of the risky asset. Given this decrease, some programme traders will sell the risky assets in order to adjust their portfolio. This leads to a further price reduction, which now will be lower than the normal level, that is $S_{t}<S_{t}^{*}$. Thus, the action of programme traders leads to potential gains for liquidity providers, such as market makers and market timers (see Grossman, 1988). These latter could buy the assets since their actual price is now lower than their normal price. In this way, liquidity providers have a stabilizing function. Such ability to exploit gains from excess volatility of price dynamics depends on some parameters, for example, the cost of capital, transaction costs $(c)$ and also the information about how many agents are using a dynamic hedging strategy. If liquidity providers commit insufficient capital, then their stabilizing function will be reduced.

Let us indicate by $\rho(c, \Im) \in[0,1]$ a parameter measuring market liquidity, which is related to the action of liquidity providers (market timers and market makers) in response to programme traders' demand, which may affect the price dynamics. $\rho(c, \Im)$ is a function of the transaction costs $c$ and $\Im \in \Re_{+}$, which captures the effects of other variables on market liquidity, such as the lack of information on hedging activity and the cost of capital. We take $\Im$ and $c$ as exogenous variables. We make the following assumptions about the behaviour of $\rho(c, \Im)$ :

\section{Assumption 4}

(a) $\partial \rho / \partial c>0, \quad \partial \rho / \partial \Im>0$;

(b) $\lim _{c \rightarrow \infty} \rho(c, \Im)=1$;

(c) $\lim _{(c, \Im) \rightarrow(0,0)} \rho(c, \Im)=0$;

(d) $\lim _{c \rightarrow 0} \rho(c, \Im) \geqslant 0$ and $\lim _{c \rightarrow 0} \rho(c, \Im)>0$ as long as $\Im>0$. 
Assumption 4(a) implies that an increase in the transaction costs and in the exogenous parameter $\Im$ reduces the liquidity of the market; 4(b) implies that as transaction costs diverge towards infinity, the market is completely illiquid; 4(c) implies that the market is perfectly liquid if the supply of capital is perfectly elastic, information is perfect and if there are no transaction costs; 4(d) implies that if transaction costs are vanishing small, then the market will still be illiquid, where the size of illiquidity depends on the size of the exogenous variable $\Im$.

Let us add the demand of programme traders to the demand of reference traders. This can be done since reference traders are supposed to be unaware of the presence of programme traders, otherwise they would condition $D\left(t, F_{t}, S_{t}\right)$ on the programme traders' strategy. Using the market clearing condition, we have that the equilibrium price has to satisfy the following condition:

$$
D\left(t, F_{t}, S_{t}\right)+\rho(c, \Im) \Psi\left(S_{t}, \sigma, c\right)=1,
$$

where, given the input volatility $\sigma, \Psi\left(S_{t}, \sigma, c\right)$ is given by (6). According to Assumption 4 , for $(c, \Im) \rightarrow(0,0)$ the action of the programme traders has a negligible effect on the price dynamics of the risky asset. Thus, each deviation of prices from their normal level will be eliminated through the action of market timers. On the other side, if the supply of capital is not perfectly elastic or market timers do not have perfect information, then market timers cannot completely eliminate the effect of the programme traders' action (see Grossman, 1988). Therefore, $\rho(c, \Im)=0$ denotes a perfectly liquid market, that is, liquidity providers are able to neutralize programme traders' demand and thus there is no deviation of the asset price from its fundamental value; as long as $\rho(c, \Im)>0$ the market for the underlying asset is only finitely liquid, and for $\rho(c, \Im)=1$ it becomes completely illiquid. Notice that an increase in the transaction costs increases the weight of portfolio insurance in the aggregate demand.

Now we can prove the main result.

Proposition 1 Given the input volatility $\sigma$, the diffusion process governing the dynamics of the asset price is

$$
d S_{t}=\mu_{S}\left(S_{t}, t ; \sigma, c\right) d t+\sigma_{S}\left(S_{t}, t ; \sigma, c\right) d W_{t},
$$

where

$$
\sigma_{S}\left(S_{t}, t ; \sigma, c\right)=-D_{F} \Theta \sigma_{F}\left(F_{t}, t\right) ;
$$




$$
\begin{aligned}
\mu_{S}\left(S_{t}, t ; \sigma, c\right)= & -\Theta\left\{D_{t}+D_{F} \mu_{F}\left(F_{t}, t\right)+\frac{1}{2} D_{F F}\left(\sigma_{F}\left(F_{t}, t\right)\right)^{2}\right. \\
& -D_{S F} D_{F} \Theta\left(\sigma_{F}\left(F_{t}, t\right)\right)^{2} \\
& +\frac{1}{2}\left[D_{S S}+\rho(c, \Im) \Psi_{S S}\left(S_{t}, \sigma, c\right)\right] \\
& \left.\times\left[D_{F} \Theta \sigma_{F}\left(F_{t}, t\right)\right]^{2}\right\}
\end{aligned}
$$

and where $\Theta=1 /\left(D_{S}+\rho(c, \Im) \Psi_{S}\left(S_{t}, \sigma, c\right)\right)$.

Proof We follow the steps of the derivation of the dynamics of the normal price outlined in 'The basic model' section. Using the implicit function theorem we can write the market clearing condition (9) as $S_{t}=\xi\left(t, F_{t}\right)$; using Ito's Lemma, we have that the dynamics of $S_{t}$ are described by the stochastic differential equation

$$
d S_{t}=\xi_{t} d t+\xi_{F} d F_{t}+\frac{1}{2} \xi_{F F}\left(d F_{t}\right)^{2}
$$

For each value of $f$ and $t, \quad s=\xi(t, f)$ and $D(t, f, \xi(t, f))+\rho(c, \Im) \times$ $\Psi(\xi(t, f), \sigma, c)=1$ hold. Applying the implicit function theorem we obtain that $\xi_{t}=-D_{t} /\left(D_{S}+\rho \Psi_{S}\right), \xi_{F}=-D_{F} /\left(D_{S}+\rho \Psi_{S}\right)$ and $\xi_{F F}=-\frac{1}{D_{S}+\rho \Psi_{S}}\left[D_{F F}-\right.$ $\left.2 D_{S F} \frac{D_{F}}{D_{S}+\rho \Psi_{S}}+\left(D_{S S}+\rho \Psi_{S S}\right)\left(\frac{D_{F}}{D_{S}+\rho \Psi_{S}}\right)^{2}\right]$. Substituting these results into (12) and using (2) the result stated in the proposition follows. Obviously, we need $\sigma_{S}\left(S_{t}, t ; \sigma, c\right) \geqslant 0$, and this is true for appropriate values of $d$ in Assumption 1.

Expression (11) gives the endogenously determined market volatility, depending, among other parameters, on the input volatility $\sigma$ used by programme traders to compute the hedging strategy. From expression (11) in Proposition 1 we observe that the larger is gamma $\left(\Delta_{S}(S, \sigma, K, \tau)\right)$, the larger is the price volatility. Since gamma indicates how often a position must be rehedged on average in order to maintain a delta-neutral position, the higher is its average value, the more frequently an adjustment occurs. At the same time, $\tilde{h}(\eta, c)$ indicates the change in the size of the adjustment, given a change in the asset price. This latter depends on the properties of the adjustment hazard function and on the size of the deterministic component of the confidence level $c$. Such component depends directly on the size of the transaction costs. Thus, the larger the transaction costs, the lower $\tilde{h}(\eta, c)$. On the other side, the larger are transaction costs, the less liquid is the market, and therefore the weight of 
126

the demand of portfolio insurance in the aggregate demand of the risky asset increases. Thus, the effect of a change in transaction costs on the volatility of the underlying asset is ambiguous a priori. Given Assumptions 2 and 4 we have that if transaction costs are infinitely high, then $\lim _{c \rightarrow \infty} \rho(c, \Im) \Psi_{S}(S, \sigma, c)=0$ and so on average no portfolio adjustment occurs and there will be no feedback effect, that is $S_{t}=S_{t}^{*}$. On the other side, if transaction costs are vanishing small, then, since $\lim _{c \rightarrow 0} \Psi_{S}(S, \sigma, c)=\tilde{\Gamma}(S, \sigma)$, we are back to dynamic hedging strategies where the liquidity of the market $\rho(0, \Im)$ depends just on the size of $\Im$, and thus we are back to a situation like the one studied by Frey and Stremme (1997).

We can summarize our results as follows. The volatility of the underlying asset is larger, the larger are the frequency of adjustment $\left(\Delta_{S}(S, \sigma, K, \tau)\right)$ and/or the size of adjustment $(\tilde{h}(\eta, c))$. The volatility depends also on $\rho(c, \Im)$. The more illiquid is the market, the higher is the influence of the hedging activity of the programme traders on the dynamics of the underlying asset price, that is, the stronger is the feedback effect.

Notice that as long as $\rho(c, \Im)>0$ and $c<\infty$ the dynamics of $S_{t}$ are different from the dynamics of the normal price $S_{t}^{*}$. In particular, comparing (11) with (5) we observe that there exists an excess volatility which is due to the hedging activity of programme traders. The size of the excess volatility depends on the liquidity of the market $(\rho(c, \Im))$ and on the aggregate characteristics of the programme traders $\left(\Psi_{S}(S, \sigma, c)\right)$. But since expression (11) for the volatility $\sigma_{S}\left(S_{t}, t ; \sigma, c\right)$ still depends on the input volatility $\sigma$, consistency requires that the input volatility $\sigma$ be equal to the endogenously determined market volatility $\sigma_{S}\left(S_{t}, t ; \sigma, c\right)$. In other words, we have to solve a fixed-point problem, that is, find a $\sigma$ such that $\sigma_{S}\left(S_{t}, t ; \sigma, c\right)=\sigma$.

In solving the fixed-point problem, we make use of the following Lemma.

Lemma (i) $\Psi_{S}$ is a bounded function of $\sigma$; (ii) for $\sigma \geqslant \sigma_{0}$, with $0<\sigma_{0}<\infty$, $\partial \Psi_{S} / \partial \sigma$, is a bounded function of $\sigma$.

Proof (i) The following equalities hold, because of the definition of $\Delta$ and Assumption 3:

$$
\begin{aligned}
\Psi_{S} & =\iint \frac{\partial \Delta}{\partial S} \tilde{h}(.) v_{1}(K, \tau) d K d \tau \\
& =-\iint \frac{K}{S} \frac{\partial \Delta}{\partial K} \tilde{h}(.) v_{1}(K, \tau) d K d \tau \\
& =\iint \Delta \frac{\partial}{\partial K}\left(\frac{K}{S} \tilde{h}(.) v_{1}\right) d K d \tau
\end{aligned}
$$


since $v_{1}$ has a compact support. In view of Assumption 2 and since $0 \leqslant \Delta \leqslant 1$, we get:

$$
\left|\Psi_{S}\right| \leqslant \iint\left|\frac{\partial}{\partial K}\left(\frac{K}{S} \tilde{h}(.) v_{1}\right)\right| d K d \tau
$$

that is, $\Psi_{S}$ is a bounded function of $\sigma$.
(ii) Recall that $\partial \Delta / \partial S=N^{\prime}\left(d_{1}\right) / \sigma S \sqrt{\tau}$, where $d_{1}=\frac{\ln \left(\frac{S}{K}\right)+\left(r+\frac{1}{2} \sigma^{2}\right) \tau}{\sigma \sqrt{\tau}}$.

Then, $\frac{\partial}{\partial \sigma}\left(\frac{N^{\prime}\left(d_{1}\right)}{\sigma S \sqrt{\tau}}\right) \leqslant N^{\prime \prime}\left(d_{1}\right) \frac{1}{\sigma S \sqrt{\tau}}-\frac{N^{\prime}\left(d_{1}\right)}{\sigma^{2} S \sqrt{\tau}} \leqslant \frac{1}{\sigma_{0} S \sqrt{\tau}}+\frac{1}{\sigma_{0}^{2} S \sqrt{\tau}}$; in view of Assumption 2, $\partial \Psi_{S} / \partial \sigma$ is a bounded function of $\sigma$ for $\sigma>\sigma_{0}$.

We are now able to state and proof the following Proposition.

Proposition 2 Under Assumptions 1-4, there exists a solution to the fixedpoint problem $\sigma_{S}\left(S_{t}, t ; \sigma, c\right)=\sigma$, provided that $\rho(c, \Im)$ is sufficiently small, where $\sigma_{S}\left(S_{t}, t ; \sigma, c\right)$ is given by (11).

Proof Let us put $M(\sigma)=\sigma_{S}(S, t ; \sigma, c)=-\frac{D_{F} \sigma_{F}\left(F_{t}, t\right)}{D_{S}+\rho(c, \Im) \Psi_{S}(S, \sigma, c)}$. We have to show that $|\partial M(\sigma) / \partial \sigma| \leqslant M<1$, with $0 \leqslant M \leqslant 1$, in order to apply the contraction mapping theorem.

We have that

$$
\left|\frac{\partial M(\sigma)}{\partial \sigma}\right|=\left|\frac{D_{F} \sigma_{F} \rho \frac{\partial}{\partial \sigma} \Psi_{S}}{\left(D_{S}+\rho \Psi_{S}\right)^{2}}\right|
$$

Let us first consider the denominator. If $|\rho|<\varepsilon$ we get, for some $\tilde{e}$ :

$$
\left|D_{S}+\rho \Psi_{S}\right| \geqslant\left|D_{S}\right|-|\rho|\left|\Psi_{S}\right| \geqslant d-\varepsilon \tilde{e} \geqslant \frac{d}{2}
$$

provided that $\varepsilon \leqslant \mathrm{d} / 2 \tilde{e}$. (13) holds because of Assumption 1(b) and the Lemma. Let us consider the numerator. We get, with suitable constants $\widetilde{\widetilde{e}}, J$ :

$$
\left|-D_{F} \sigma_{F} \rho \frac{\partial}{\partial \sigma} \Psi_{S}\right|=\left|D_{F} \sigma_{F}\right||\rho|\left|\frac{\partial}{\partial \sigma} \Psi_{S}\right| \leqslant D_{F} \sigma_{F} \mid \rho \widetilde{\widetilde{e}} \leqslant J \varepsilon .
$$

It holds because of the Lemma. Therefore, $\left|\frac{\partial M(\sigma)}{\partial \sigma}\right| \leqslant \frac{4 J \varepsilon}{d^{2}}=M \leqslant 1$, which holds for $\varepsilon \leqslant \min \left\{\frac{d}{2 \tilde{e}}, \frac{d^{2}}{4 J}\right\}$.

Finally, we have to check that $\sigma_{S}\left(S_{t}, t ; \sigma, c\right)=M(\sigma) \geqslant \sigma_{0}$, where $0<\sigma_{0}<\infty$, as required by the Lemma. Since $\frac{-D_{F} \sigma_{F}}{D_{S}+\rho \Psi_{S}}=\frac{D_{F} \sigma_{F}}{-D_{S}-\rho \Psi_{S}} \geqslant \frac{D_{F} \sigma_{F}}{-D_{S}}>0$, we can put $\sigma_{0}=\frac{D_{F} \sigma_{F}}{-D_{S}}$, which completes the proof.

Proposition 2, which ensures existence and consistency of the equilibrium, puts a restriction on the market weight $\rho(c, \Im)$ of programme traders and thus makes the notion of "finitely liquid" market more precise. 
128

Moreover, Proposition 2 specifies the extent to which it is appropriate to use Black-Scholes strategies for hedging purposes. In practice, most traders base their strategies on the classical Black-Scholes formula, which assumes constant volatility $\sigma$, while we recognized that the correct value is $\sigma_{S}(S, t ; \sigma, c)$, incorporating the feedback effects due to the interaction between the degree of market liquidity, the size and the frequency of adjustment and transaction costs. In the presence of feedback effects, Black-Scholes strategies based on the assumption of a constant volatility produce a tracking error that is almost surely non-zero. El Karoui et al. (1998) show how to derive a formula for the tracking error, which measures the difference between the actual and the theoretical value of a self-financing hedge portfolio for a European call calculated from the Black-Scholes formula with constant volatility. Proposition 2 gives us an insight about the behaviour of the tracking error: clearly, for $\rho(c, \Im)$ sufficiently small, as required in Proposition 2, the tracking error vanishes.

\section{Conclusion}

We extend the analysis of feedback effects of dynamic hedging strategies on the underlying asset to the case of fixed costs of transactions in finitely liquid markets. Our results are in keeping with the literature on increased market volatility from dynamic hedging strategies. However, in this paper we specify in which way markets are finitely liquid when transaction costs are introduced and the role of transaction costs in determining the size and the frequency of adjustment. Our results can be of interest for applications, since we provide a quantitative estimate of the increased volatility with transaction costs, which establishes a precise interaction between the degree of market liquidity, the size and frequency of adjustment, and transaction costs. We show that the action of programme traders leads to an excess volatility of the asset price, the size of which depends on the average size of adjustment and on the average gamma. The former depends on the properties of the adjustment hazard function and on the size of transaction costs, whereas the latter indicates how often, on average, a position must be rehedged in order to maintain a delta neutral position. Finally, we show how the fixed-point problem for the volatility of the asset price can be solved, to conclude that from a practical viewpoint it may be reasonable to use Black-Scholes strategies based on $\sigma_{S}\left(S_{t}, t ; \sigma, c\right)$ for hedging purposes provided that the difference between $\sigma_{S}\left(S_{t}, t ; \sigma, c\right)$ and $\sigma$ is sufficiently small.

\section{Acknowledgements}

The authors thank two anonymous referees for their invaluable comments and suggestions. 


\section{References}

Bensaid, B., Lesne, J.-P., Pages, J.-P. and Scheinkman, J. (1992) 'Derivative asset pricing with transaction costs', Mathematical Finance 2: 63-86.

Brennan, M.J. and Schwartz, E.S. (1989) 'Portfolio insurance and financial market equilibrium', Journal of Business 62: 455-472.

Caballero, R.J. and Engel, E.M.R.A. (1993) 'Microeconomic adjustment hazards and aggregate dynamics', Quarterly Journal of Economics 108: 359-383.

Caballero, R.J. and Engel, E.M.R.A. (1999) 'Explaining investment dynamics in U.S. manufacturing: A generalized (S,s) approach', Econometrica 67: 783-826.

Cetin, U., Jarrow, R. and Protter, P. (2004) 'Liquidity risk and arbitrage pricing theory', Finance and Stochastics 8: 311-341.

Cetin, U., Jarrow, R., Protter, P. and Warachka, M. (2006) 'Pricing options in an extended Black Scholes economy with illiquidity: Theory and empirical evidence', The Review of Financial Studies 19: 493-529.

Cetin, U., Soner, H.M. and Touzi, N. (2007) 'Option Hedging for Small Investors under Liquidity Costs', http://people.sabanciuniv.edu/msoner/cetin-soner-touzi.pdf.

Constantinides, G. and Zariphopoulou, T. (1999) 'Bounds on prices of contingent claims in an intertemporal economy with proportional transaction costs and general preferences', Finance and Stochastics 3: 345-369.

Constantinides, G. and Zariphopoulou, T. (2001) 'Bounds on derivative prices in an intertemporal setting with proportional transaction costs and multiple securities', Mathematical Finance 11: 331-346.

Davis, M.H.A., Panas, V.G. and Zariphopoulou, T. (1993) 'European option pricing with transaction costs', SIAM Journal of Control and Optimization 31: 470-493.

Dixit, A. (1993) The Art of Smooth Pasting, Harwood Academic Publishers: Switzerland.

Donaldson, R.G. and Uhlig, H. (1993) 'The impact of large portfolio insurers on asset price', The Journal of Finance XLVIII: 1943-1955.

Dumas, B., Fleming, J. and Whaley, R.E. (1998) 'Implied volatility functions: Empirical tests', The Journal of Finance 53: 2059-2106.

El Karoui, N., Jeanblanc-Picqué, M. and Shreve, S. (1998) 'Robustness of the Black and Scholes formula', Mathematical Finance 8: 93-126.

Frey, R. (1998) 'Perfect option replication for a large trader', Finance and Stochastics 2: $115-142$.

Frey, R. and Stremme, A. (1997) 'Market volatility and feedback effects from dynamic hedging', Mathematical Finance 7: 351-374.

Gennotte, G. and Leland, H. (1990) 'Market liquidity, hedging and crashes', American Economic Review 80: 999-1021.

Gibson, R., Habib, M.A. and Ziegler, A. (2008) 'Financial markets, reinsurance and the bearing of national catastrophic risk', mimeo.

Grossman, S.J. (1988) 'An analysis of the implications for stock and futures price volatility of program trading and dynamic hedging strategies', Journal of Business 61: 275-298.

Hasbrouck, J. (2009) 'Trading costs and returns for U.S. equities: Estimating effective costs from daily data', Journal of Finance 64: 1445-1477.

Henrotte, P. (1993) Transaction costs and duplication strategies, working paper, Stanford University.

Hoggard, T., Whalley, A.E. and Wilmott, P. (1994) 'Hedging option portfolios in the presence of transaction costs', Advances in Futures and Options Research 8: 222-239.

Jarrow, R. (1992) 'Market manipulation, bubbles, corners and short squeezes', Journal of Financial and Quantitative Analysis 27: 311-336. 
Jarrow, R. (1994) 'Derivative securities markets, market manipulation and option pricing theory', Journal of Financial and Quantitative Analysis 29: 241-261.

Kabanov, Y.M. and Safarian, M.M. (1997) 'On Leland's strategy of option pricing with transaction costs', Finance and Stochastics 1: 239-250.

Leland, H. (1985) 'Option pricing and replication with transaction costs', Journal of Finance $\mathbf{1 5}$ 1283-1301.

Le Roy, S. (1989) 'Efficient capital markets and martingales', Journal of Economic Literature 27: 1583-1622.

LeRoy, S. and Porter, R. (1981) 'The present-value relation: Tests based on implied variance bounds', Econometrica 44: 555-574.

Platen, E. and Schweizer, M. (1994) On smile and skewness, Statistics Research Report 027-94, Australian National University, Canberra.

Platen, E. and Schweizer, M. (1998) 'On feedback effects from hedging derivatives', Mathematical Finance 8: 67-84.

Rubinstein, M. (1985) 'Non-parametric tests of alternative option pricing models using all reported trades and quotes on the 30 most active CBOE option classes from August, 23, 1976 through August, 31, 1978', Journal of Finance 40: 455-480.

Schönbucher, P. and Wilmott, P. (2000) 'The feedback effect of hedging in illiquid markets', SIAM Journal of Applied Mathematics 61: 232-272.

Shiller, R.J. (1981) 'Do stock prices move too much to be justified by subsequent changes in dividend?' American Economic Review 76: 421-435.

Shiller, R.J. (2003) 'From efficient markets theory to behavioral finance', Journal of Economic Perspectives 17: 83-104.

Sircar, K.R. and Papanicolaou, G. (1998) 'General Black-Scholes models accounting for increased market volatility from hedging strategies', Applied Mathematical Finance 5: 45-82.

Whalley, A.E. and Wilmott, P. (1993) Option pricing with transaction costs, MFG working paper, Oxford.

Whalley, A.E. and Wilmott, P. (1997) 'An asymptotic analysis of an optimal hedging model for option pricing with transaction costs', Mathematical Finance 7: 307-324.

Wilmott, P. (2000) Paul Wilmott on Quantitative Finance, New York, NY: John Wiley \& Sons, LTD.

\section{About the Authors}

Elettra Agliardi is Full Professor in Economics, since 1 November 2000, University of Bologna. She obtained an MPhil in Economics and a PhD in Economics from the University of Cambridge; a $\mathrm{PhD}$ in Economics (with special mention), European University Institute - Prize "Obiettivo Europa" for the best PhD dissertation. Her academic positions in U.K.: (i) 1 October 1990 - 30 September 1992 Research Officer, at Department of Applied Economics (DAE), University of Cambridge, U.K.; (ii) 1 October 1990 - 30 September 1995, College Lecturer in Economics and Fellow (Title A), Churchill College, University of Cambridge, U.K. Her academic positions in Italy: (i) 1 November 1992 - 31 October 1995, Associate Professor at Faculty of Economics, University of Cassino; (ii) 1 November 1995 - 31 October 2000, Associate Professor in Economics at Faculty of Economics, University of Bologna; and 
since 1 November 2000 is Full Professor in Economics, at Faculty of Economics (Rimini Campus), University of Bologna. Her other University activities include Director of the PhD Programme in Economics, University of Bologna, 2001-2003; Director of Studies of the Laurea Magistralis "Economics and Market Policy" since 2008; Member of the Editorial Board of the Review of Economic Analysis; Member of the Scientific Board of the PhD Programme in Economics, University of Bologna since 1999; Referee Member of the National Research Assessment CIVR 2005; Referee Member of the National Research Programmes MIUR since 2003; Referee Member of the National Programme "Rientro dei Cervelli", MIUR 2008. Her research interests include the derivative pricing, real options, corporate finance and liquidation policies, bank capital requirements and deposit insurance, executive compensation and financial options, effects of taxation on liquidation policies.

Rainer Andergassen is an assistant professor at the Faculty of Economics in Rimini, University of Bologna. He obtained a Bachelor's degree with distinction from the University of Bologna (1997), a Master's Degree in Economics with distinction from the University College London (2000) and a $\mathrm{PhD}$ in Economics from the University of Siena (2002). Before joining the University of Bologna as an assistant professor in January 2005, he held postdoctoral positions from 2001 to 2003 and from 2004 to 2005 at the same University. Among his research interests are the dynamics of technical progress and economic growth, inequality and economic development, executive compensation and incentives, and real option analysis. He has authored or co-authored several papers in refereed international journals, including Journal of Economic Dynamics and Control, Journal of Economic Behavior and Organization, Economics Letters, Journal of Macroeconomics (http://www2.dse.unibo.it/anderga/). 